\title{
Engineering semiconductor nanowires for photodetection: from visible to terahertz
}

Hannah J. Joyce, Jack Alexander-Webber, Kun Peng, Michael B. Johnston, Patrick Parkinson, et al.

Hannah J. Joyce, Jack Alexander-Webber, Kun Peng, Michael B. Johnston, Patrick Parkinson, H. Hoe Tan, C. Jagadish, "Engineering semiconductor nanowires for photodetection: from visible to terahertz," Proc. SPIE 10729, Optical Sensing, Imaging, and Photon Counting: From X-Rays to $\mathrm{THz}$, 1072909 (18 September 2018); doi: 10.1117/12.2320715

SPIE Event: SPIE Nanoscience + Engineering, 2018, San Diego, California, United States 


\title{
Engineering semiconductor nanowires for photodetection: from visible to terahertz
}

\author{
$\underline{\text { Hannah J. Joyce }}^{* a}$, Jack Alexander-Webber ${ }^{\mathrm{a}}$, Kun Peng ${ }^{\mathrm{b}}$, Michael B. Johnston ${ }^{\mathrm{b}}$, Patrick Parkinson ${ }^{\mathrm{c}}$, \\ H. Hoe Tan ${ }^{\mathrm{d}}$, C. Jagadish ${ }^{\mathrm{d}}$ \\ ${ }^{a}$ Department of Engineering, University of Cambridge, $9 \mathrm{JJ}$ Thomson Avenue \\ Cambridge CB3 0FA, United Kingdom; \\ ${ }^{\mathrm{b}}$ Department of Physics, University of Oxford, Clarendon Laboratory, Parks Road, \\ Oxford OX1 3PU, United Kingdom; \\ ${ }^{\mathrm{c}}$ School of Physics \& Astronomy and the Photon Science Institute, University of Manchester, \\ Manchester M13 9PL, United Kingdom; \\ ${ }^{\mathrm{d}}$ Department of Electronic Materials Engineering, Research School of Physics and Engineering, \\ The Australian National University, Canberra ACT 2601, Australia
}

\begin{abstract}
III-V semiconductor nanowires combine the properties of III-V materials with the unique advantages of the nanowire geometry, allowing efficient room temperature photodetection across a wide range of photon energies, from a few $\mathrm{eV}$ down to meV. For example, due to their nanoscale size, these show great promise as sub-wavelength terahertz (THz) detectors for near-field imaging or detecting elements within a highly integrated on-chip $\mathrm{THz}$ spectrometer. We discuss recent advances in engineering a number of sensitive photonic devices based on III-V nanowires, including InAs nanowires with tunable photoresponse, $\mathrm{THz}$ polarisers and $\mathrm{THz}$ detectors.
\end{abstract}

Keywords: Nanowire, III-V, semiconductor, terahertz, photoresponse

\section{INTRODUCTION}

III-V semiconductor nanowires combine the properties of III-V materials, such as their direct bandgaps and high charge carrier mobilities, with the unique advantages of the quasi-one-dimensional nanowire geometry, such as efficient lateral strain relaxation, optical waveguiding, enhanced light trapping, and reduced cost and materials consumption relative to conventional planar devices. These properties are advantageous for photodetection, and indeed efficient nanowire-based photodetectors have been demonstrated across a wide range of photon energies, from a few $\mathrm{eV}$ down to $\mathrm{meV}$, all operating at room temperature. Due to their nanoscale size, nanowires show particular promise as sub-wavelength terahertz $(\mathrm{THz})$ detectors for near-field imaging or detecting elements within a highly integrated "on-chip" THz spectrometer. The sensitivity and nature of photodetection can be tailored by tuning the optical and electronic properties of the nanowires, for instance by passivating their surface states and tuning their charge carrier lifetimes. We discuss recent advances in engineering a number of sensitive photonic devices based on III-V nanowires, including InAs nanowire-based field effect transistors (FETs) with tunable photoresponse, ${ }^{1} \mathrm{THz}$ polarisers based on GaAs nanowires, ${ }^{2}$ and sub-wavelength $\mathrm{THz}$ detectors. ${ }^{3,4}$

*hannah.joyce@eng.cam.ac.uk; phone +44 1223748379

Optical Sensing, Imaging, and Photon Counting: From X-Rays to THz, edited by Oleg Mitrofanov,

Chee Hing Tan, José Luis Pau Vizcaíno, Manijeh Razeghi, Proc. of SPIE Vol. 10729, 1072909

(C) 2018 SPIE · CCC code: $0277-786 X / 18 / \$ 18 \cdot$ doi: 10.1117/12.2320715 


\section{GROWTH OF NANOWIRES}

InAs, GaAs and GaAs/AlGaAs core-shell nanowires were grown by metalorganic chemical vapour deposition (MOCVD) using Au nanoparticles to drive nanowire growth. Prior to growth (111)B substrates were functionalised with poly-L-lysine then treated with a solution of monodisperse colloidal Au nanoparticles. A range of nanoparticle diameters $(20,30,50,80,100$ or $150 \mathrm{~nm})$ were studied. Each Au nanoparticle drives the growth of a single nanowire, and the wetting of the nanowire tip by the nanoparticle determines the diameter of the nanowire. ${ }^{5}$

Nanowires were grown in an Aixtron 200/4 horizontal flow MOCVD reactor at a pressure of 100 mbar and a total gas flow rate of $15 \mathrm{slm}$. Group III precursors trimethylgallium, trimethylaluminium and trimethylindium and the group V precursor arsine were employed. Substrates were first annealed under group V overpressure to desorb surface contaminants. After cooling to the desired growth temperature the group III precursor flow was introduced to the reactor to initiate nanowire growth. To achieve high quality nanowires we optimised a number of growth parameters including substrate temperature and the gas flow rates of the group III and V precursors.

The InAs nanowires used for fabrication of field-effect transistors were grown at a temperature of $500{ }^{\circ} \mathrm{C}$ and a V/III ratio of 2.9. These InAs nanowires featured wurtzite crystal structures and hexagonal cross-sections with $\{1-100\}$ sidefacets. ${ }^{6,7}$ A scanning electron microscopy (SEM) image of such InAs nanowires is shown in Figure 1a.

The GaAs nanowires used for the THz polarisers were grown on (100)-oriented substrates and grew along two [111]B directions which occur at angles of $35.3^{\circ}$ to the (100) substrate plane. When projected on the substrate plane the nanowires appear parallel, as seen in the plan view SEM image of Figure 1b. These zinc-blende GaAs nanowires were grown by a two-temperature process as previously published ${ }^{8}$ in which a short $(60 \mathrm{~s})$ high temperature $\left(450{ }^{\circ} \mathrm{C}\right)$ nucleation step is used to enhance the yield of straight [111]B-oriented nanowires, and thereafter a longer growth step at a lower temperature $\left(375{ }^{\circ} \mathrm{C}\right.$ to $\left.425{ }^{\circ} \mathrm{C}\right)$ reduces parasitic radial growth. ${ }^{8} \mathrm{~A}$ high V/III ratio of 46 and a moderately fast growth rate were employed to reduce the incidence of twin defects. ${ }^{9,10}$

Photoconductive $\mathrm{THz}$ detectors were fabricated from GaAs/AlGaAs core-shell nanowires. The GaAs cores of the $\mathrm{GaAs} / \mathrm{AlGaAs}$ core-shell nanowires were either grown by the two-temperature process that gives purely zinc-blende nanowires, or by a single temperature process that results in twin defects in the nanowire core. AlGaAs shells were grown at $750{ }^{\circ} \mathrm{C}$ under conditions that enhance the radial growth rate over the axial growth rate. ${ }^{11}$

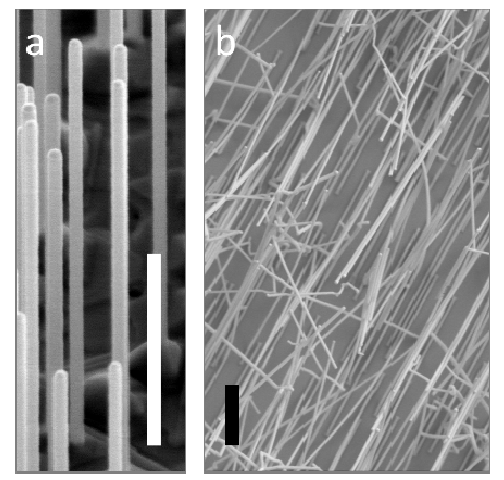

Figure 1. SEM images of (a) InAs nanowires at a tilt of $40^{\circ}$ and (b) aligned GaAs nanowires in plan view.

\section{PHOTORESPONSE OF NANOWIRE-BASED FIELD EFFECT TRANSISTORS}

Wurtzite InAs nanowires were mechanically transferred to a doped $\mathrm{Si}$ wafer with a $300 \mathrm{~nm}$ thick $\mathrm{SiO}_{2}$ layer. The wafer functioned as a back gate and the $\mathrm{SiO}_{2}$ layer functioned as the dielectric. To achieve a $1 \mu \mathrm{m}$ channel length, electron beam lithography was used to define source and drain regions, followed by passivation of the nanowire surface in $2 \%$ aqueous $\left(\mathrm{NH}_{4}\right)_{2} \mathrm{~S}$ solution at $40{ }^{\circ} \mathrm{C}$ for $10 \mathrm{~min}$, then sputter deposition of $70 \mathrm{~nm} \mathrm{Ni}$, followed by lift-off. Measurements were carried out using a probe station connected to a Keithley 4200-SCS semiconductor characterisation system. All measurements were performed at room temperature. As surface-adsorbed $\mathrm{H}_{2} \mathrm{O}$ is known to affect the transport properties of wurtzite InAs nanowires, ${ }^{12}$ we performed the measurements under ambient humidity conditions and under vacuum. 
To measure the photoresponse, illumination was applied using a $3200 \mathrm{~K}$ halogen lamp with a power density of $30 \mathrm{~mW}$ $\mathrm{cm}^{-2}$.

All the InAs nanowire-based FETs exhibited n-type conduction, whereby more positive gate voltages switch conduction on whereas more negative gate voltages turned off the conduction. The transfer curves obtained from the unpassivated devices showed large hysteresis between the upwards and downwards gate voltage sweeps. ${ }^{13}$ The threshold voltage shifts to a more positive voltage on the downwards sweep. This indicates the presence of a trap states that charge with electrons as the gate bias is increased to more positive values, and discharge as the gate bias is swept back to negative voltages.

Figure 2 illustrates the transient response of a InAs nanowire-based FET to photoexcitation. We applied a back gate voltage of $30 \mathrm{~V}$ to switch the FET on and a source-drain bias of $10 \mathrm{mV}$. Upon illumination, the drain current dropped by 3 orders of magnitude and even after illumination was ceased, the drain current and conductivity did not recover for several hours. The conductivity could be restored by the application of a negative or zero bias to the gate, which releases trapped electrons. ${ }^{1}$ The long-lived negative photoconductivity has strong potential for application in non-volatile optical memory devices, but is undesirable for photodetection and energy harvesting applications.

Negative photoconductivity is more pronounced for nanowires with higher surface area-to-volume ratios (smaller diameter), indicating that the traps are associated with surface states. To mitigate the effect of the surface states we investigated passivation of the nanowires. A number of devices were passivated with a $90 \mathrm{~nm}$-thick layer of $\mathrm{Al}_{2} \mathrm{O}_{3}$ deposited by atomic layer deposition (ALD) using a Cambridge NanoTech ALD system at $120{ }^{\circ} \mathrm{C}$ using trimethylaluminium and $\mathrm{H}_{2} \mathrm{O}$ precursors. After passivation with $\mathrm{Al}_{2} \mathrm{O}_{3}$ the nanowire FETs no longer showed negative photoconductivity and instead showed a positive photoresponse, that is, an increase in drain current due to photoexcitation. Passivation also significantly reduced hysteresis and increased the field effect mobility by almost an order of magnitude. ${ }^{1}$

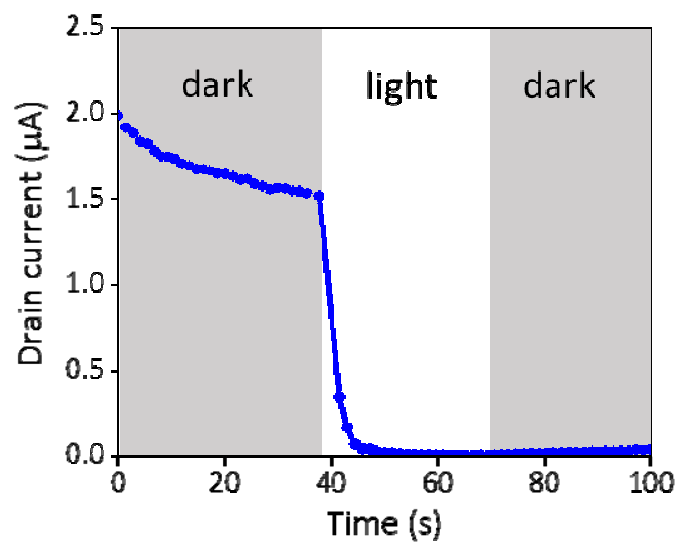

Figure 2. Drain current as a function of time after application of a source-drain bias of $10 \mathrm{mV}$, both in the dark and under illumination. The drain current drops after illumination with a halogen lamp, corresponding to negative photoconductivity. The drain current remains low even after the illumination is removed.

\section{PHOTOCONDUCTIVE TERAHERTZ DETECTORS BASED ON SINGLE NANOWIRES}

Nanowires were dispersed on z-cut quartz substrates that are transparent to THz radiation. Contacts were patterned on opposite ends of GaAs/AlGaAs core-shell nanowires using direct write laser lithography. To remove the native oxide from the nanowires a chemical etch in $4 \% \mathrm{HCl}$ was performed. The Ti/Au $(10 \mathrm{~nm} / 300 \mathrm{~nm})$ contacts were deposited by electron beam evaporation and lift-off. Figure 3 is a schematic diagram of the photoconductive nanowire THz detector.

The single-nanowire photoconductive $\mathrm{THz}$ detectors were tested in THz time domain spectroscopy (TDS) system. A single-cycle pulse of $\mathrm{THz}$ radiation was generated using a semi-insulating GaAs photoconductive switch with a $400 \mu \mathrm{m}$ 
gap between the electrodes. As a reference for comparison with our single-nanowire devices, we used a photoconductive receiver fabricated from ion-implanted InP with a bow-tie antenna structure.

These GaAs/AlGaAs core-shell nanowires exhibit long photoconductivity lifetimes (> $100 \mathrm{ps}) .{ }^{14}$ Therefore the photoconductive receiver functions in the integrating regime in which the measured photocurrent is related to the timeintegrated THz electric field. ${ }^{15}$ The detection bandwidth of the nanowire-based photodetectors was 0.1 to $0.6 \mathrm{THz}{ }^{3}$ Devices fabricated with twin-free nanowires gave higher signal-to-noise ratios than devices that used twinned GaAs nanowires. The higher signal-to-noise ratio was related to the higher mobility and photoconductivity in the twin-free GaAs nanowires. ${ }^{16}$

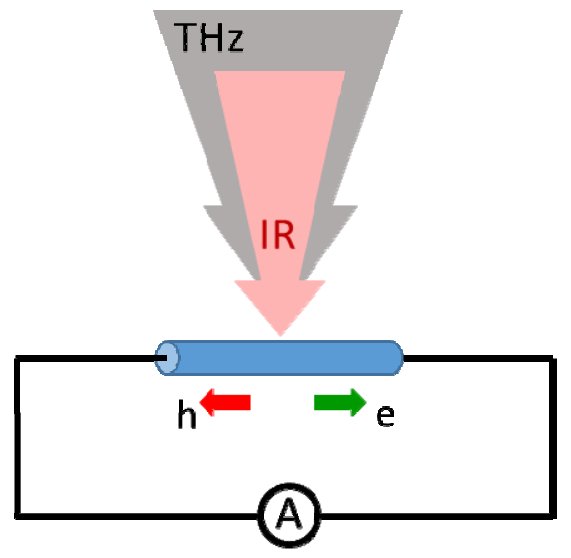

Figure 3. Schematic diagram of a single-nanowire photoconductive $\mathrm{THz}$ detector.

\section{NANOWIRE-BASED TERAHERTZ POLARISERS}

$\mathrm{THz}$ communication systems require practical, fast and switchable $\mathrm{THz}$ modulators. GaAs nanowires exhibit high electron mobilities, short photoconductivity lifetimes, a highly polarisation-sensitive and ultrafast response to photoexcitation, and highly anisotropic conductivity, which makes them ideal components for an optically switchable $\mathrm{THz}$ polarisation modulators. We fabricated polarisers based on aligned GaAs nanowires embedded in Parylene C polymer. A $5 \mu \mathrm{m}$ layer of Parylene $\mathrm{C}$ was deposited on top of the aligned GaAs nanowires grown as described in Section 2. Parylene $\mathrm{C}$ coats the nanowires conformally and is a mechanically robust matrix. The films of aligned nanowires embedded in Parylene $\mathrm{C}$ were then peeled from the substrates and characterised via optical pump-terahertz probe spectroscopy. A half-wave plate was placed in the optical pump beam path to vary the polarisation of the optical pump.

Photoexcitation renders the nanowires conductive, and these polarisers become opaque to $\mathrm{THz}$ radiation when photoexcited with an $800 \mathrm{~nm}$ pulse (35 fs duration) with polarisation parallel to the nanowire axis. The ultrashort photoconductivity lifetime $\left(<1\right.$ ps rise and $<5$ ps fall times) of the nanowires ${ }^{17}$ means that these polarisers can be switched at high frequencies. In order to gain the highest modulation depth we laminated together several nanowirepolymer thin films. These polarisers exhibited a modulation depth of $-8 \mathrm{~dB}$, an extinction of over $13 \%$ and a dynamic range of $-9 \mathrm{~dB}$.

\section{CONCLUSIONS}

We have demonstrated a number of sensitive photonic devices based on III-V nanowires. InAs nanowire-based FETs with tunable photoresponse were achieved by surface passivation with ALD-deposited alumina. ${ }^{1}$ Photoconductive $\mathrm{THz}$ detectors based on GaAs/AlGaAs core-shell nanowires exhibit strong sensitivity despite their sub-wavelength size, making them suitable for on-chip and near-field THz TDS systems. ${ }^{3}$ THz polarisers based on GaAs nanowires achieve THz modulation with a switching time of less than $5 \mathrm{ps}$ and a modulation depth of $-8 \mathrm{~dB}{ }^{2}$ 


\section{ACKNOWLEDGEMENTS}

The authors gratefully acknowledge financial support from the European Research Council (ERC Starting Grant ACrossWire), the Engineering and Physical Sciences Research Council (UK), the Australian Research Council, and the Australian National Fabrication Facility (ANFF). J. A. Alexander-Webber and H. J. Joyce especially thank the Royal Commission for the Exhibition of 1851 for their research fellowships.

\section{REFERENCES}

[1] Alexander-Webber, J. A., Groschner, C. K., Sagade, A. A., Tainter, G., Gonzalez-Zalba, M. F., Pietro, R. Di, Wong-Leung, J., Tan, H. H., Jagadish, C., Hofmann, S. and Joyce, H. J., "Engineering the Photoresponse of InAs Nanowires," ACS Appl. Mater. Interfaces 9, 43993-44000 (2017).

[2] Baig, S. A., Boland, J. L., Damry, D. A., Tan, H. H., Jagadish, C., Joyce, H. J. and Johnston, M. B., “An Ultrafast Switchable Terahertz Polarization Modulator Based on,” Nano Lett. 17, 2603-2610 (2017).

[3] Peng, K., Parkinson, P., Fu, L., Gao, Q., Jiang, N., Guo, Y.-N., Wang, F., Joyce, H. J., Boland, J. L., Tan, H. H., Jagadish, C. and Johnston, M. B., "Single nanowire photoconductive terahertz detectors," Nano Lett. 15(1), 206210 (2015).

[4] Peng, K., Parkinson, P., Boland, J. L., Gao, Q., Wenas, Y. C., Davies, C. L., Li, Z., Fu, L., Johnston, M. B., Tan, H. H. and Jagadish, C., "Broadband Phase-Sensitive Single InP Nanowire Photoconductive Terahertz Detectors," Nano Lett. 16(8), 4925-4931 (2016).

[5] Jiang, N., Wong-Leung, J., Joyce, H. J., Gao, Q., Tan, H. H. and Jagadish, C., “Understanding the true shape of Au-catalyzed GaAs nanowires," Nano Lett. 14(10), 5865-5872 (2014).

[6] Joyce, H. J., Wong-Leung, J., Gao, Q., Hoe Tan, H. and Jagadish, C., "Phase perfection in zinc blende and wurtzite III-V nanowires using basic growth parameters," Nano Lett. 10(3), 908-915 (2010).

[7] Joyce, H. J., Gao, Q., Wong-Leung, J., Kim, Y., Tan, H. H. and Jagadish, C., "Tailoring GaAs, InAs, and InGaAs nanowires for optoelectronic device applications," IEEE J. Sel. Top. Quantum Electron. 17(4), 766-778 (2011).

[8] Joyce, H. J., Gao, Q., Tan, H. H., Jagadish, C., Kim, Y., Zhang, X., Guo, Y. and Zou, J., “Twin-free uniform epitaxial GaAs nanowires grown by a two-temperature process," Nano Lett. 7(4), 921-926 (2007).

[9] Joyce, H. J., Gao, Q., Tan, H. H., Jagadish, C., Kim, Y., Fickenscher, M. A., Perera, S., Hoang, T. B., Smith, L. M., Jackson, H. E., Yarrison-Rice, J. M., Zhang, X. and Zou, J., "High purity GaAs nanowires free of planar defects: Growth and characterization," Adv. Funct. Mater. 18(23), 3794-3800 (2008).

[10] Joyce, H. J., Gao, Q., Tan, H. H., Jagadish, C., Kim, Y., Fickenscher, M. A., Perera, S., Hoang, T. B., Smith, L. M., Jackson, H. E., Yarrison-Rice, J. M., Zhang, X. and Zou, J., "Unexpected benefits of rapid growth rate for III-V nanowires," Nano Lett. 9(2) (2009).

[11] Jiang, N., Parkinson, P., Gao, Q., Breuer, S., Tan, H. H., Wong-Leung, J. and Jagadish, C., "Long minority carrier lifetime in Au-catalyzed GaAs/ $\mathrm{Al}_{x} \mathrm{Ga}_{1-\mathrm{x}} \mathrm{As}$ core-shell nanowires," Appl. Phys. Lett. 101(2), 23111 (2012).

[12] Ullah, A. R., Joyce, H. J., Tan, H. H., Jagadish, C. and Micolich, A. P., "The influence of atmosphere on the performance of pure-phase WZ and ZB InAs nanowire transistors," Nanotechnology 28(45) (2017).

[13] Carrad, D. J., Mostert, A. B., Ullah, A. R., Burke, A. M., Joyce, H. J., Tan, H. H., Jagadish, C., Krogstrup, P., Nygård, J., Meredith, P. and Micolich, A. P., "Hybrid Nanowire Ion-to-Electron Transducers for Integrated Bioelectronic Circuitry,” Nano Lett. 17(2) (2017).

[14] Joyce, H. J., Parkinson, P., Jiang, N., Docherty, C. J., Gao, Q., Tan, H. H., Jagadish, C., Herz, L. M. and Johnston, M. B., "Electron mobilities approaching bulk limits in 'surface-free' GaAs nanowires," Nano Lett. 14(10), 5989-5994 (2014).

[15] Castro-Camus, E., Fu, L., Lloyd-Hughes, J., Tan, H. H., Jagadish, C. and Johnston, M. B., "Photoconductive response correction for detectors of terahertz radiation," J. Appl. Phys. 104(5) (2008). 
[16] Parkinson, P., Joyce, H. J., Gao, Q., Tan, H. H., Zhang, X., Zou, J., Jagadish, C., Herz, L. M. and Johnston, M. B., "Carrier lifetime and mobility enhancement in nearly defect-free core-shell nanowires measured using timeresolved terahertz spectroscopy,” Nano Lett. 9(9), 3349-3353 (2009).

[17] Joyce, H. J., Docherty, C. J., Gao, Q., Tan, H. H., Jagadish, C., Lloyd-Hughes, J., Herz, L. M. and Johnston, M. B., "Electronic properties of GaAs, InAs and InP nanowires studied by terahertz spectroscopy.," Nanotechnology 24(21), 214006 (2013). 